\title{
On timelike supersymmetric solutions of Abelian gauged 5-dimensional supergravity
}

\author{
Samuele Chimento \\ Instituto de Física Teórica UAM/CSIC, \\ C/ Nicolás Cabrera, 13-15, C.U. Cantoblanco, E-28049 Madrid, Spain \\ E-mail: samuele.chimento@csic.es
}

ABSTRACT: We consider 5-dimensional gauged $\mathcal{N}=1$ supergravity coupled to Abelian vector multiplets, and we look for supersymmetric solutions for which the 4-dimensional Kähler base space admits a holomorphic isometry. Taking advantage of this isometry, we are able to find several supersymmetric solutions for the ST $\left[2, n_{v}+1\right]$ special geometric model with arbitrarily many vector multiplets. Among these there are three families of solutions with $n_{v}+2$ independent parameters, which for one of the families can be seen to correspond to $n_{v}+1$ electric charges and one angular momentum. These solutions generalize the ones recently found for minimal gauged supergravity in JHEP 1704 (2017) 017 and include in particular the general supersymmetric asymptotically- $\mathrm{AdS}_{5}$ black holes of Gutowski and Reall, analogous black hole solutions with non-compact horizon, the three near horizon geometries themselves, and the singular static solutions of Behrndt, Chamseddine and Sabra.

KeYwords: Black Holes, Black Holes in String Theory, Supergravity Models

ARXiv EPRINT: 1705.01903 


\section{Contents}

1 Introduction 1

2 Abelian gauged $\mathcal{N}=1, d=5$ supergravity 3

2.1 Timelike supersymmetric solutions 4

3 Timelike supersymmetric solutions of Abelian gauged $\mathcal{N}=1, d=5$ $\begin{array}{lr}\text { supergravity with one additional isometry } & 7\end{array}$

$\begin{array}{lll}3.1 \text { Summary } & 11\end{array}$

4 Solutions $\quad 12$

$\begin{array}{lll}4.1 \text { Ansatz } & 12\end{array}$

$\begin{array}{ll}4.2 & \text { Solutions for the } \mathrm{ST}\left[2, n_{v}+1\right] \text { model } \\ \end{array}$

$\begin{array}{lll}4.3 & \text { Case } 1 & 16\end{array}$

$\begin{array}{lll}\text { 4.3.1 Supersymmetric black holes } & 18\end{array}$

$\begin{array}{lll}\text { 4.3.2 } & \text { Conserved charges } & 19\end{array}$

$\begin{array}{lll}\text { 4.3.3 Static solutions } & 20\end{array}$

$\begin{array}{lll}4.4 & \text { Case } 2 & 22\end{array}$

5 Conclusions 23

\section{Introduction}

Exact solutions of supergravity theories have been and continue to be instrumental in gaining new insights into string theory and related areas of research. In particular asymptotically anti-de Sitter solutions, which occur naturally in gauged supergravity, are interesting from the point of view of the AdS/CFT correspondence, since in that context they can be viewed as gravitational duals of strongly coupled quantum systems living on the AdS boundary.

Symmetry has always been one of the main tools in the search for exact solutions of gravity theories, since requiring the invariance of the solution under some symmetry transformation can dramatically simplify the usually formidable task of solving the equations of motion.

In the supergravity setting it is natural to look for solutions with some unbroken supersymmetry. This implies that the bosonic equations of motion are related through the Killing Spinor Identities [1], reducing the problem of solving them to that of solving just a small subset plus the first order supersymmetry equations. Besides the simplification in the equations they entail, another motivation for the study of supersymmetric solutions in AdS, specifically supersymmetric black holes, is that it should be possible to determine their entropy by counting their microstates through the AdS/CFT correspondence (this is still an open problem for rotating supersymmetric black holes in $\mathrm{AdS}_{5}$, see e.g. [2-4]). 
However, while assuming unbroken supersymmetry makes the problem more tractable, it is usually not enough to find explicit solutions, and one has to make some additional assumptions or to impose a specific ansatz in order to solve the equations. ${ }^{1}$

An approach that has proven to be very successful in ungauged 5-dimensional supergravity, with or without vector multiplets, is to assume that the 4-dimensional base space, which for that theory has to be hyperKähler, admits one triholomorphic isometry. In this case the base space has a Gibbons-Hawking metric [6,7], and it turns out that the solutions can be completely characterized in terms of a small number of building blocks, namely harmonic functions on 3-dimensional flat space $[8,9]$. The same ansatz has also been effective for $\mathcal{N}=1, d=5$ supergravity with vector multiplets and non-Abelian gaugings [10], but without Fayet-Iliopoulos terms, in which case the base space is again a 4-dimensional hyperKähler space.

Recently [11] a similar ansatz was applied to the case of minimal $d=5$ gauged supergravity, where a $\mathrm{U}(1)$ subgroup of the $\mathrm{SU}(2)$ R-symmetry group is gauged by adding a Fayet-Iliopoulos term to the bosonic action. In this case the base space is just Kähler, instead of hyperKähler, and the ansatz consists in assuming that it admits a holomorphic isometry. The metric of the base space can then be written in terms of two functions [12] in a form that generalizes the Gibbons-Hawking metrics, and the problem of finding supersymmetric solutions is reduced to that of solving a system of fourth order differential equations for these two functions plus a third one.

The aim of this paper is to apply the same ansatz in the case of $\mathcal{N}=1, d=5$ supergravity with vector multiplets and Abelian Fayet-Iliopoulos gaugings, where a U(1) subgroup of the $\mathrm{SU}(2) \mathrm{R}$-symmetry group is gauged with a linear combination of the vector fields of the theory, in which case the base space is again Kähler.

The paper is organized as follows. Section 2 consists in a quick review of the theory and the conditions to impose on the fields in order to obtain (timelike) supersymmetric solutions. In section 3 we adapt the supersymmetry equations to the assumption that the 4-dimensional Kähler base space of the solution admits a holomorphic isometry, after writing the general form for a metric of this kind. In section 4, after making some additional assumptions, we find several supersymmetric solutions for the special geometric model ST $\left[2, n_{v}+1\right]$ with an arbitrary number $n_{v}$ of vector multiplets. Among these are three general classes of superficially asymptotically-AdS solutions ${ }^{2}$ that can be seen as a generalization in the presence of vector multiplets of solutions found recently for pure gauged supergravity [11]. They are studied in some detail in subsection 4.3, where the conserved charges are computed for one of the families, and it is shown that they include as particular cases black holes with compact or non-compact horizon, as well as static singular solutions. In subsection 4.4 we give the explicit expression of the fields for supersymmetric black holes not included in the solutions of subsection 4.3, despite being very similar to a subcase of them. We conclude in section 5 with some final remarks.

\footnotetext{
${ }^{1}$ For a comprehensive review of supersymmetric solutions of supergravity theories with many references see, e.g. ref. [5].

${ }^{2}$ By superficially asymptotically-AdS we mean that the metric components approach those of AdS in an appropriate limit, which however does not guarantee that the solutions are globally asymptotically-AdS.
} 


\section{Abelian gauged $\mathcal{N}=1, d=5$ supergravity}

In this section we give a brief description of the bosonic sector of a general theory of $\mathcal{N}=1, d=5$ supergravity coupled to $n_{v}$ vector multiplets in which a $\mathrm{U}(1)$ subgroup of the $\mathrm{SU}(2) \mathrm{R}$-symmetry group has been gauged by the addition of Fayet-Iliopoulos (FI) terms. The U(1) subgroup to be gauged and the gauge vector used in the gauging are determined by the tensor $\mathrm{P}_{I}{ }^{r}$, as we are going to explain. ${ }^{3}$ Our conventions are those in refs. $[13,14]$ which are those of ref. [15] with minor modifications.

The supergravity multiplet is constituted by the graviton $e_{\mu}^{a}$, the gravitino $\psi_{\mu}^{i}$ and the graviphoton $A_{\mu}$. All the spinors are symplectic Majorana spinors and carry a fundamental $\mathrm{SU}(2) \mathrm{R}$-symmetry index. The $n_{v}$ vector multiplets, labeled by $x=1, \ldots, n_{v}$ consist of a real vector field $A_{\mu}^{x}$, a real scalar $\phi^{x}$ and a gaugino $\lambda^{i x}$.

It is convenient to combine the matter vector fields $A_{\mu}^{x}$ with the graviphoton $A_{\mu} \equiv A_{\mu}^{0}$ into a vector $\left(A_{\mu}^{I}\right)=\left(A_{\mu}^{0}, A_{\mu}^{i}\right)$. It is also convenient to define a vector of functions of the scalars $h^{I}(\phi) . \mathcal{N}=1, d=5$ supersymmetry requires that these $n_{v}+1$ functions of the $n_{v}$ scalars satisfy a constraint of the form

$$
C_{I J K} h^{I}(\phi) h^{J}(\phi) h^{K}(\phi)=1,
$$

where the constant symmetric tensor $C_{I J K}$ completely characterizes the ungauged theory and the Special Real geometry of the scalar manifold. In particular, the kinetic matrix of the vector fields $a_{I J}(\phi)$ and the metric of the scalar manifold $g_{x y}(\phi)$ can be derived from it as follows: first, we define

$$
h_{I} \equiv C_{I J K} h^{J} h^{K}, \quad \Rightarrow \quad h^{I} h_{I}=1
$$

and

$$
h_{x}^{I} \equiv-\sqrt{3} h^{I}{ }_{, x} \equiv-\sqrt{3} \frac{\partial h^{I}}{\partial \phi^{x}}, \quad h_{I x} \equiv+\sqrt{3} h_{I, x}, \quad \Rightarrow \quad h_{I} h_{x}^{I}=h^{I} h_{I x}=0 .
$$

Then, $a_{I J}$ is defined implicitly by the relations

$$
h_{I}=a_{I J} h^{I}, \quad h_{I x}=a_{I J} h_{x}^{J} .
$$

It can be checked that

$$
a_{I J}=-2 C_{I J K} h^{K}+3 h_{I} h_{J} .
$$

The metric of the scalar manifold $g_{x y}(\phi)$, which we will use to raise and lower $x, y$ indices is (proportional to) the pullback of $a_{I J}$

$$
g_{x y} \equiv a_{I J} h_{x}^{I} h_{y}^{J}=-2 C_{I J K} h_{x}^{I} h_{y}^{J} h^{K} .
$$

We will use the completeness relation

$$
h_{I} h^{J}+g^{x y} h_{x I} h_{y}^{J}=\delta_{I}^{J} .
$$

\footnotetext{
${ }^{3}$ Although its origin is different, it can be understood as a particular example of embedding tensor.
} 
The FI gauging of any model of $\mathcal{N}=1, d=5$ supergravity coupled to vector multiplets is completely determined by the choice of $\mathrm{P}_{I}{ }^{r}$, where $r=1,2,3$ is a $\mathfrak{s u}(2)$ index. In the Abelian case, this tensor can be factorized as follows:

$$
\mathrm{P}_{I}^{r}=g c_{I} d^{r} \equiv g_{I} d^{r}
$$

where $g$ is the gauge coupling constant, $d^{r}$ (which we can normalize $d^{r} d^{r}=1$ ) chooses a direction in $S^{3}$ or, equivalently, a $\mathfrak{u}(1) \subset \mathfrak{s u}(2)$ to be gauged and $c_{I}$ (also normalized $c_{I} c_{I}=1$ ) dictates which linear combination of the vector fields, $c_{I} A^{I}{ }_{\mu}$, acts as gauge field. $g_{I}=g c_{I}$ is a convenient combination of constants that we will use. We will not make any specific choices for the time being.

The bosonic action is given in terms of $a_{I J}, g_{x y}$ and $C_{I J K}$ and $\mathrm{P}_{I}^{r}$

$$
\begin{aligned}
S= & \int d^{5} x \sqrt{g}\left\{R+\frac{1}{2} g_{x y} \partial_{\mu} \phi^{x} \partial^{\mu} \phi^{y}-V(\phi)-\frac{1}{4} a_{I J} F^{I \mu \nu} F_{\mu \nu}^{J}\right. \\
& \left.+\frac{C_{I J K} \varepsilon^{\mu \nu \rho \sigma \alpha}}{12 \sqrt{3} \sqrt{g}} F^{I}{ }_{\mu \nu} F^{J}{ }_{\rho \sigma} A^{K}{ }_{\alpha}\right\},
\end{aligned}
$$

where the Abelian vector field strengths are $F^{I}{ }_{\mu \nu}=2 \partial_{[\mu} A^{I}{ }_{\nu]}$ and the scalar potential $V(\phi)$ is given by

$$
V(\phi)=-\left(4 h^{I} h^{J}-2 g^{x y} h_{x}^{I} h_{y}^{J}\right) \mathrm{P}_{I}^{r} \mathrm{P}_{J}^{r}=-4 C^{I J K} h_{I} \mathrm{P}_{J}^{r} \mathrm{P}_{K}^{r} .
$$

The equations of motion for the bosonic fields are

$$
\begin{aligned}
G_{\mu \nu}-\frac{1}{2} a_{I J}\left(F_{\mu}^{I}{ }_{\mu} F^{J}{ }_{\nu \rho}-\frac{1}{4} g_{\mu \nu} F^{I \rho \sigma} F^{J}{ }_{\rho \sigma}\right) & \\
+\frac{1}{2} g_{x y}\left(\partial_{\mu} \phi^{x} \partial_{\nu} \phi^{y}-\frac{1}{2} g_{\mu \nu} \partial_{\rho} \phi^{x} \partial^{\rho} \phi^{y}\right)+\frac{1}{2} g_{\mu \nu} V & =0, \\
\nabla_{\nu}\left(a_{I J} F^{J \nu \mu}\right)+\frac{1}{4 \sqrt{3}} \frac{\varepsilon^{\mu \nu \rho \sigma \alpha}}{\sqrt{g}} C_{I J K} F^{J}{ }_{\nu \rho} F^{k}{ }_{\sigma \alpha} & =0, \\
\nabla_{\mu} \partial^{\mu} \phi^{x}+\frac{1}{4} g^{x y} \partial_{y} a_{I J} F^{I \rho \sigma} F^{J}{ }_{\rho \sigma}+g^{x y} \partial_{y} V & =0 .
\end{aligned}
$$

\subsection{Timelike supersymmetric solutions}

The general form of the solutions of these theories admitting a timelike Killing spinor ${ }^{4}$ was found in refs. [16-18]. In what follows we are going to review it using the notation and results of ref. [14] in which general non-Abelian gaugings were considered, ${ }^{5}$ but restricting to Abelian FI gaugings.

The building blocks of the timelike supersymmetric solutions are the scalar function $\hat{f}$, the 4-dimensional spatial metric $h_{m n}{ }^{6}{ }^{6}$ an antiselfdual almost hypercomplex structure $\hat{\Phi}^{(r)}{ }_{m n},{ }^{7}$ a 1-form $\hat{\omega}_{\underline{m}}$, the 1-form potentials $\hat{A}^{I} \underline{m}$ and the scalars of the theory combined

\footnotetext{
${ }^{4} \mathrm{~A}$ timelike (commuting) spinor $\epsilon^{i}$ is, by definition, such that the real vector bilinear constructed from it $i V_{\mu} \sim \bar{\epsilon}_{i} \gamma_{\mu} \epsilon^{i}$ is timelike.

${ }^{5}$ Even more general gaugings were considered in [19] with the inclusion of tensor multiplets.

${ }^{6} m, n, p=1, \cdots, 4$ will be tangent space indices and $\underline{m}, \underline{n}, \underline{p}=1, \cdots, 4$ will be curved indices. We are going to denote with hats all objects that naturally live in this 4-dimensional space.

${ }^{7}$ That is: the 2-forms $\hat{\Phi}^{(r)}{ }_{m n} r, s, t=1,2,3$ satisfy

$$
\begin{aligned}
\hat{\Phi}^{(r) m n} & =-\frac{1}{2} \varepsilon^{m n p q} \hat{\Phi}_{p q}^{(r)}, \quad \text { or } \quad \hat{\Phi}^{(r)}=-\star_{4} \hat{\Phi}^{(r)}, \\
\hat{\Phi}^{(r) m}{ }_{n} \hat{\Phi}^{(s) n}{ }_{p} & =-\delta^{r s} \delta^{m}{ }_{p}+\varepsilon^{r s t} \hat{\Phi}^{(t) m}{ }_{p} .
\end{aligned}
$$


into the functions $h^{I}(\phi)$. All these fields are defined on the 4-dimensional spatial manifold usually called "base space". They are time-independent and must satisfy a number of conditions:

1. The antiselfdual almost hypercomplex structure $\hat{\Phi}^{(r)}{ }_{m n}$, the 1-form potentials $\hat{A}^{I} \underline{m}$ and the base space metric $h_{\underline{m n}}$ (through its Levi-Civita connection) satisfy the equation

$$
\hat{\nabla}_{m} \hat{\Phi}^{(r)}{ }_{n p}+\varepsilon^{r s t} \hat{A}_{m}^{I} \mathrm{P}_{I}{ }^{s} \hat{\Phi}^{(t)}{ }_{n p}=0 .
$$

2. The selfdual part of the spatial vector field strengths $\hat{F}^{I} \equiv d \hat{A}^{I}$ must be related to the function $\hat{f}$, the 1 -form $\hat{\omega}$ and the scalars of the theory by

$$
h_{I} \hat{F}^{I+}=\frac{2}{\sqrt{3}}(\hat{f} d \hat{\omega})^{+},
$$

3. while the antiselfdual part is related to the almost hypercomplex structure by ${ }^{8}$

$$
\hat{F}^{I-}=-2 \hat{f}^{-1} C^{I J K} h_{J} \mathrm{P}_{K}^{r} \hat{\Phi}^{(r)} .
$$

4. Finally, all the building blocks are related by the equation

$$
\hat{\nabla}^{2}\left(h_{I} / \hat{f}\right)-\frac{1}{6} C_{I J K} \hat{F}^{J} \cdot \hat{\star} \hat{F}^{K}+\frac{1}{2 \sqrt{3}}\left(a_{I K}-2 C_{I J K} h^{J}\right) \hat{F}^{K} \cdot(\hat{f} d \hat{\omega})^{-}=0,
$$

where the dots indicate standard contraction of all the indices of the tensors.

Once the building blocks that satisfy the above conditions have been found, the physical 5-dimensional fields can be built out of them ${ }^{9}$ as follows:

1. The 5-dimensional (conformastationary) metric is given by

$$
d s^{2}=\hat{f}^{2}(d t+\hat{\omega})^{2}-\hat{f}^{-1} h_{\underline{m n}} d x^{m} d x^{n} .
$$

2. The complete 5 -dimensional vector fields are given by

$$
A^{I}=-\sqrt{3} h^{I} e^{0}+\hat{A}^{I}, \quad \text { where } e^{0} \equiv \hat{f}(d t+\hat{\omega}),
$$

so that the spatial components are

$$
A_{\underline{m}}^{I}=\hat{A}_{\underline{m}}^{I}-\sqrt{3} h^{I} \hat{f} \hat{\omega}_{\underline{m}},
$$

and the 5 -dimensional field strength is

$$
F^{I}=-\sqrt{3} d\left(h^{I} e^{0}\right)+\hat{F}^{I} .
$$

${ }^{8}$ In this equation the indices of $C^{I J K}$ have been raised using the inverse metric $a^{I J}$ and one has the useful relations

$$
C^{I J K} h_{K}=h^{I} h^{J}-\frac{1}{2} g^{x y} h_{x}^{I} h_{y}^{J}=\frac{3}{2} h^{I} h^{J}-\frac{1}{2} a^{I J} .
$$

${ }^{9}$ In the ungauged case the above conditions determine the quotients $h_{I} / \hat{f}$ from which $\hat{f}$ can be found by using the condition eq. (2.1). 
3. The scalar fields $\phi^{x}$ can be obtained by inverting the functions $h_{I}(\phi)$ or $h^{I}(\phi)$. A parametrization which is always available is

$$
\phi^{x}=h_{x} / h_{0}
$$

As it has already been observed in refs. $[16,18]$ choosing $d^{r}=\delta^{r}{ }_{1}$ we see that eq. (2.16) gives us additional information: it splits into

$$
\begin{aligned}
\hat{\nabla}_{m} \hat{\Phi}^{(1)}{ }_{n p} & =0, \\
\hat{\nabla}_{m} \hat{\Phi}^{(2)}{ }_{n p} & =\hat{P}_{m} \hat{\Phi}^{(3)}{ }_{n p}, \\
\hat{\nabla}_{m} \hat{\Phi}^{(3)}{ }_{n p} & =-\hat{P}_{m} \hat{\Phi}^{(2)}{ }_{n p},
\end{aligned}
$$

where we have defined

$$
\hat{P}_{m} \equiv g_{I} \hat{A}_{m}^{I} .
$$

The first equation means that the metric $h_{\underline{m n}}$ is Kähler with respect to the complex structure $\hat{J}_{m n} \equiv \hat{\Phi}^{(1)}{ }_{m n}$. Taking this fact into account, ${ }^{10}$ the integrability condition of the other two equations is ${ }^{11}$

$$
\hat{\mathfrak{R}}_{m n}=-2 \hat{\nabla}_{[m} \hat{P}_{n]}=-g_{I} \hat{F}_{m n}^{I} .
$$

This equation must be read as a constraint on the 1 -form potentials $\hat{A}_{\underline{m}}^{I}$ posed by the choice of base space metric.

Eq. (2.19) takes a simpler form as well:

$$
\hat{F}^{I-}=-2 \hat{f}^{-1} C^{I J K} h_{J} g_{K} \hat{J}, \quad \Rightarrow \quad\left\{\begin{array}{l}
g_{I} \hat{F}^{I-}=\frac{1}{2} \hat{f}^{-1} V(\phi) \hat{J}, \\
h_{I} \hat{F}^{I-}=-2 \hat{f}^{-1} g_{I} h^{I} \hat{J} .
\end{array}\right.
$$

Tracing the first of these equations and eq. (2.34) with $\hat{J}^{m n}$ one finds a relation between the Ricci scalar of the base space metric, the scalar potential and the function $\hat{f}$ :

$$
\hat{R}=-2 V / \hat{f} .
$$

${ }^{10}$ We use the integrability condition of eq. (2.26)

$$
\hat{R}_{m n p q}=\hat{R}_{m n r s} \hat{J}_{p}^{r} \hat{J}_{q}^{s},
$$

which leads to the relation between the Ricci and Riemann tensors

$$
\hat{R}_{m n}=-\frac{1}{2} \hat{R}_{m p r q} \hat{J}^{r q} \hat{J}_{n}^{p} .
$$

The Ricci 2-form, defined as

$$
\hat{\mathfrak{R}}_{m n} \equiv \hat{R}_{m p} \hat{J}_{n}^{p}
$$

is, therefore, related to the Riemann tensor by

$$
\hat{\mathfrak{R}}_{m n}=\frac{1}{2} \hat{R}_{m n p q} \hat{J}^{p q} .
$$

\footnotetext{
${ }^{11}$ If $P_{m}$ vanishes (for instance, in the ungauged case), then we have a covariantly constant hyper-Kähler structure and, then, the base space is hyperKähler.
} 
The last equation to be simplified by our choice is eq. (2.20). Substituting in it eq. (2.35) and using eqs. (2.18) and the completeness relation eq. (2.7) one finds

$$
\hat{\nabla}^{2}\left(h_{I} / \hat{f}\right)-\frac{1}{6} C_{I J K} \hat{F}^{J} \cdot \hat{\star} \hat{F}^{K}+\frac{1}{\sqrt{3}} g_{I} \hat{J} \cdot(d \hat{\omega})=0 .
$$

In order to make progress one has to start making specific assumptions about the base space metric. In the ungauged $[8,13]$ and the non-Abelian gauged cases [10] it has proven very useful to assume that the base space metric has an additional isometry because, then, it depends on a very small number of independent functions. Recently the same assumption was made for pure gauged supergravity [11], where the base space can be a general Kähler metric, allowing to reduce the problem of finding supersymmetric solutions to a system of fourth order differential equations for three functions. In what follows we are going to make the same assumption for the case at hand, in which vector multiplets are present, in the attempt to simplify the task of finding supersymmetric solutions.

\section{Timelike supersymmetric solutions of Abelian gauged $\mathcal{N}=1, d=5$ supergravity with one additional isometry}

Any four-dimensional Kähler metric with one holomorphic isometry can be written locally as [12]:

$$
d s^{2}=H^{-1}(d z+\chi)^{2}+H\left\{\left(d x^{2}\right)^{2}+W^{2}\left[\left(d x^{1}\right)^{2}+\left(d x^{3}\right)^{2}\right]\right\},
$$

with the functions $H$ and $W$, and the 1-form $\chi$, depending only on the three coordinates $x^{i}$ and satisfying the constraints:

$$
\begin{aligned}
& (d \chi)_{\underline{12}}=\partial_{\underline{3}} H, \\
& (d \chi)_{\underline{23}}=\partial_{\underline{1}} H, \\
& (d \chi)_{\underline{31}}=\partial_{\underline{2}}\left(W^{2} H\right),
\end{aligned}
$$

whose integrability condition is

$$
\mathfrak{D}^{2} H \equiv \partial_{\underline{1}}^{2} H+\partial_{\underline{2}}^{2}\left(W^{2} H\right)+\partial_{\underline{3}}^{2} H=0 .
$$

In a frame defined by the Vierbein

$$
\begin{aligned}
e^{\sharp} & =H^{-1 / 2}(d z+\chi), \\
e^{2} & =H^{1 / 2} d x^{2}, \\
e^{1,3} & =H^{1 / 2} W d x^{1,3},
\end{aligned}
$$

the conserved complex structure is given by

$$
\left(\hat{J}_{m n}\right)=\left(\begin{array}{rr}
0_{2 \times 2} & \mathbb{1}_{2 \times 2} \\
-\mathbb{1}_{2 \times 2} & 0_{2 \times 2}
\end{array}\right) .
$$

The Ricci tensor and Ricci scalar of the 4-dimensional metric can be expressed in terms of the functions $H$ and $W^{2}$ in a compact form,

$$
\hat{R}_{m n}=\hat{\nabla}_{m} \hat{\nabla}_{n} \log W+\hat{J}_{m}{ }^{p} \hat{J}_{n}^{q} \hat{\nabla}_{p} \hat{\nabla}_{q} \log W, \quad \hat{R}=\hat{\nabla}^{2} \log W^{2},
$$


where the 4-dimensional Laplacian acts on $z$-independent functions as

$$
\hat{\nabla}^{2} f=H^{-1} \bar{\nabla}^{2} f=\frac{1}{H W^{2}}\left[\partial_{\underline{1}}^{2} f+\partial_{\underline{2}}\left(W^{2} \partial_{\underline{2}} f\right)+\partial_{\underline{3}}^{2} f\right]
$$

and $\bar{\nabla}^{2}$ is the Laplacian operator associated with the 3-dimensional metric

$$
d s_{3}^{2}=\left(d x^{2}\right)^{2}+W^{2}\left[\left(d x^{1}\right)^{2}+\left(d x^{3}\right)^{2}\right] .
$$

The expression for the Ricci scalar should be compared with eq. (2.36).

We will take the base space metric $h_{\underline{m n}} d x^{m} d x^{n}$ to be of the form (3.1), and we will make the identification $\hat{\Phi}^{(1)}=\hat{J}$. We can solve for $\hat{P}_{m}$ in eqs. $(2.27)$ and (2.28) if we choose a particular form for the complex structures $\hat{\Phi}^{(2,3)}$. Without loss of generality they can be chosen to be

$$
\left(\hat{\Phi}_{m n}^{(2)}\right)=\left(\begin{array}{cc}
i \sigma_{2} & 0_{2 \times 2} \\
0_{2 \times 2} & -i \sigma_{2}
\end{array}\right), \quad\left(\hat{\Phi}_{m n}^{(3)}\right)=\left(\begin{array}{cc}
0_{2 \times 2} & -i \sigma_{2} \\
-i \sigma_{2} & 0_{2 \times 2}
\end{array}\right),
$$

where $\sigma_{2}$ is the second Pauli matrix

$$
\sigma_{2}=\left(\begin{array}{rr}
0 & -i \\
i & 0
\end{array}\right)
$$

Then we find that the flat components of $P$ can be written in the compact form

$$
\hat{P}_{m}=\hat{J}_{m}^{n} \partial_{n} \log W
$$

On the other hand, recalling the definition of $\hat{P}_{m}$ eq. (2.29) we find for the gauge vector and its field strength

$$
\begin{aligned}
g_{I} \hat{A}^{I}{ }_{m} & =\hat{J}_{m}{ }^{n} \partial_{n} \log W \\
g_{I} \hat{F}^{I}{ }_{m n} & =-\Re_{m n}=-2 \hat{\nabla}_{[m \mid} \hat{\nabla}_{p} \log W \hat{J}^{p}{ }_{\mid n]} .
\end{aligned}
$$

Every (anti-)selfdual 2-form $\mathcal{F}^{ \pm}$on the four dimensional Kähler base space can be written in terms of a 1 -form living on the 3 -dimensional space $\vartheta=\vartheta_{\underline{i}} d x^{i}$ as

$$
\mathcal{F}^{ \pm}=(d z+\chi) \wedge \vartheta \pm H \star_{3} \vartheta
$$

The 2-forms we consider here are also $z$-independent and so will the components of the corresponding 1-forms be. Thus, we introduce the $z$-independent 3-dimensional 1-forms $\Lambda^{I}, \Sigma^{I}, \Omega_{ \pm}$defined by

$$
\begin{aligned}
\hat{F}^{I+} & =-\frac{1}{2}(d z+\chi) \wedge \Lambda^{I}-\frac{1}{2} H \star_{3} \Lambda^{I}, \\
\hat{F}^{I-} & =-\frac{1}{2}(d z+\chi) \wedge \Sigma^{I}+\frac{1}{2} H \star_{3} \Sigma^{I}, \\
(d \hat{\omega})^{ \pm} & =(d z+\chi) \wedge \Omega^{ \pm} \pm H \star_{3} \Omega^{ \pm},
\end{aligned}
$$


Comparing the expression of $\hat{F}^{I-}$ with eq. (2.35) and those of $h_{I} \hat{F}^{I+}$ and $(d \omega)^{+}$with eq. (2.17) we conclude that

$$
\begin{aligned}
\Sigma^{I} & =4 \hat{f}^{-1} C^{I J K} h_{J} g_{K} d x^{2}, \\
\Omega^{+} & =-\frac{\sqrt{3}}{4} \hat{f}^{-1} h_{I} \Lambda^{I}
\end{aligned}
$$

Requiring the closure of the 2-forms $\hat{F}^{I}=\hat{F}^{I+}+\hat{F}^{I-}$ one gets

$$
d\left(\Lambda^{I}+\Sigma^{I}\right)=0
$$

which means that, locally,

$$
\Lambda^{I}=d\left(K^{I} / H\right)-\Sigma^{I},
$$

for some functions $K^{I}$.

From the same condition, using eq. (3.3) and the definition of the operator $\mathfrak{D}^{2}$ in that equation, one also gets

$$
\mathfrak{D}^{2} K^{I}=2 \partial_{\underline{2}}\left(H W^{2} \Sigma_{\underline{2}}^{I}\right) .
$$

Using eq. (3.15) and its full contraction with $\hat{J}$ one finds

$$
2 g_{I} \Sigma_{\underline{2}}^{I}=\hat{\nabla}^{2} \log W^{2}, \quad g_{I} K^{I}=\partial_{\underline{2}} \log W^{2},
$$

where an integration constant reflecting the possibility of adding to the solutions $K^{I}$ of eq. (3.24) solutions of the homogeneous equation has been set to zero without loss of generality, since from (3.23) it is clear that the $K^{I}$ 's are defined up to a constant times $H$. Using these relations, eq. (3.24) contracted with $g_{I}$ is automatically satisfied, leaving $n_{V}$ independent equations.

It is convenient to rewrite $\hat{\omega}$ as

$$
\hat{\omega}=\omega_{z}(d z+\chi)+\omega, \quad \omega=\omega_{\underline{i}} d x^{i},
$$

in terms of which

$$
\Omega^{ \pm}= \pm \frac{1}{2} H^{-1}\left(\omega_{z} \star_{3} d \chi+\star_{3} d \omega\right)-\frac{1}{2} d \omega_{z} .
$$

From eqs. (3.21) and (3.23) we find that

$$
\Omega^{+}=-\frac{\sqrt{3}}{4} \frac{h_{I}}{\hat{f}}\left[d\left(K^{I} / H\right)-\Sigma^{I}\right]
$$

and, then, from eq. (3.27) we find that

$$
\Omega^{-}=-\Omega^{+}-d \omega_{z}=\frac{\sqrt{3}}{4} \frac{h_{I}}{\hat{f}}\left[d\left(K^{I} / H\right)-\Sigma^{I}\right]-d \omega_{z} .
$$

Using either of the last two equations in eq. (3.27) one gets an equation for $\omega$ :

$$
d \omega=H \star_{3} d \omega_{z}-\omega_{z} d \chi-\frac{\sqrt{3}}{2} \frac{h_{I}}{\hat{f}} H \star_{3}\left[d\left(K^{I} / H\right)-\Sigma^{I}\right] .
$$


Before calculating its integrability condition it is convenient to make a change of variables (identical to the one made in the ungauged case) to (partially) "symplecticdiagonalize" the right-hand side. Thus, we define $L_{I}$ and $M$ through

$$
\begin{aligned}
h_{I} / \hat{f} & \equiv L_{I}+\frac{1}{12} C_{I J K} K^{J} K^{K} / H, \\
\omega_{z} & \equiv M+\frac{\sqrt{3}}{4} L_{I} K^{I} / H+\frac{1}{24 \sqrt{3}} C_{I J K} K^{I} K^{J} K^{K} / H^{2} .
\end{aligned}
$$

Substituting these two expressions into eq. (3.30) and using the relation between the 1-form $\chi$ and the functions $H$ and $W$, eqs. (3.2), the equation for $\omega$ takes the form ${ }^{12}$

$$
\begin{aligned}
d \omega= & \star_{3}\left\{H d M-M d H+\frac{\sqrt{3}}{4}\left(K^{I} d L_{I}-L_{I} d K^{I}\right)\right. \\
& \left.-H\left(\omega_{z} \partial_{2} \log W^{2}-2 \sqrt{3} h^{I} g_{I} \hat{f}^{-2}\right) d x^{2}\right\},
\end{aligned}
$$

and its integrability equation is just ${ }^{13}$

$$
\begin{aligned}
& H \bar{\nabla}^{2} M-M \bar{\nabla}^{2} H+\frac{\sqrt{3}}{4}\left(K^{I} \bar{\nabla}^{2} L_{I}-L_{I} \bar{\nabla}^{2} K^{I}\right) \\
& -\frac{1}{W^{2}} \partial_{2}\left\{H W^{2}\left(\omega_{z} \partial_{\underline{2}} \log W^{2}-2 \sqrt{3} h^{I} g_{I} \hat{f}^{-2}\right)\right\}=0 .
\end{aligned}
$$

This equation can be simplified by using the equations satisfied by the functions $H$ and $K^{I}$ (3.3) and (3.24), respectively. We postpone doing this until we derive the equation for the functions $L_{I}$, which follows from eq. (2.37). First of all, observe that, with our choice of complex structure eq. (3.7)

$$
\hat{J} \cdot(d \hat{\omega})=4(d \hat{\omega})_{02}^{-}=4 \Omega_{\underline{2}}^{-}=\sqrt{3} \frac{h_{I}}{\hat{f}}\left[\partial_{\underline{2}}\left(K^{I} / H\right)-\Sigma_{\underline{2}}^{I}\right]-\partial_{\underline{2}} \omega_{z} .
$$

On the other hand, we have

$$
\begin{aligned}
\hat{\nabla}^{2}\left(h_{I} / \hat{f}\right) & =\frac{1}{H} \bar{\nabla}^{2}\left(h_{I} / \hat{f}\right), \\
\hat{F}^{J} \cdot \hat{\star} \hat{F}^{K} & =\Lambda_{m}^{J} \Lambda_{m}^{K}-\Sigma_{m}^{J} \Sigma_{m}^{K}=\partial_{m} \frac{K^{J}}{H} \partial_{m} \frac{K^{K}}{H}-2 \partial_{m} \frac{K^{(J}}{H} \Sigma_{m}^{K)}, \\
C_{I J K} H \partial_{m} \frac{K^{J}}{H} \partial_{m} \frac{K^{K}}{H} & =C_{I J K}\left[\bar{\nabla}^{2}\left(\frac{K^{J} K^{K}}{2 H}\right)+\frac{K^{J} K^{K}}{2 H^{2}} \bar{\nabla}^{2} H-\frac{K^{J} \bar{\nabla}^{2} K^{K}}{H}\right],
\end{aligned}
$$

and, using all these partial results into eq. (2.37), and (not everywhere, for the sake of simplicity) the new variables eqs. (3.31), we arrive at

$$
\begin{aligned}
\bar{\nabla}^{2} L_{I}-C_{I J K}\left[\frac{1}{12} \frac{K^{J} K^{K}}{H^{2}}\right. & \left.\bar{\nabla}^{2} H+\frac{1}{6} \frac{K^{J} \bar{\nabla}^{2} K^{K}}{H}+\frac{1}{3} H \partial_{\underline{2}}\left(K^{J} / H\right) \Sigma_{\underline{2}}^{K}\right] \\
& +g_{I} H\left\{\frac{h_{L}}{\hat{f}}\left[\partial_{\underline{2}}\left(K^{L} / H\right)-\Sigma_{\underline{2}}^{L}\right]-\frac{4}{\sqrt{3}} \partial_{\underline{2}} \omega_{z}\right\}=0 .
\end{aligned}
$$

\footnotetext{
${ }^{12}$ We have left one $\omega_{z}$ in order to get a more compact expression.

${ }^{13}$ One has $\star_{3} d \star_{3} d=\bar{\nabla}^{2}$.
} 
We can now use the relation between the 3 -dimensional Laplacian and the $\mathfrak{D}^{2}$ operator and the equations for the functions $H$ and $K^{I}$ (3.3) and (3.24)

$$
\begin{aligned}
\nabla^{2} H & =\frac{\mathfrak{D}^{2} H}{W^{2}}-\partial_{\underline{2}} H \frac{\partial_{\underline{2}} W^{2}}{W^{2}}-H \frac{\partial_{\underline{2}}^{2} W^{2}}{W^{2}}=-\partial_{\underline{2}} H \frac{\partial_{\underline{2}} W^{2}}{W^{2}}-H \frac{\partial_{\underline{2}}^{2} W^{2}}{W^{2}}, \\
\bar{\nabla}^{2} K^{I} & =\frac{\mathfrak{D}^{2} K^{I}}{W^{2}}-\partial_{\underline{2}} K^{I} \frac{\partial_{\underline{2}} W^{2}}{W^{2}}-K^{I} \frac{\partial_{\underline{2}}^{2} W^{2}}{W^{2}} \\
& =\frac{2}{W^{2}} \partial_{\underline{2}}\left(H W^{2} \Sigma_{\underline{2}}^{I}\right)-\partial_{\underline{2}} K^{I} \frac{\partial_{\underline{2}} W^{2}}{W^{2}}-K^{I} \frac{\partial_{\underline{2}}^{2} W^{2}}{W^{2}},
\end{aligned}
$$

and the equation for $L_{I}$ becomes

$$
\begin{aligned}
\bar{\nabla}^{2} L_{I}+ & \frac{C_{I J K}}{3 W^{2}} \partial_{\underline{2}}\left(W^{2} K^{J} \Sigma_{\underline{2}}^{K}-\frac{1}{4} H^{-1} K^{J} K^{K} \partial_{\underline{2}} W^{2}\right) \\
& +g_{I} H\left\{\frac{h_{L}}{\hat{f}}\left[\partial_{\underline{2}}\left(K^{L} / H\right)-\Sigma_{\underline{2}}^{L}\right]-\frac{4}{\sqrt{3}} \partial_{\underline{2}} \omega_{z}\right\}=0 .
\end{aligned}
$$

This equation, once substituted in eq. (3.33), gives

$$
\begin{aligned}
\bar{\nabla}^{2} M= & -\frac{C_{I J K}}{48 \sqrt{3} W^{2}} \partial_{\underline{2}}\left(H^{-2} K^{I} K^{J} K^{K} \partial_{\underline{2}} W^{2}\right)+\frac{C_{I J K}}{8 \sqrt{3}} H^{-1} K^{I} K^{J} \partial_{\underline{2}} \Sigma_{\underline{2}}^{K} \\
& -\frac{\sqrt{3}}{2} \Sigma_{\underline{2}}^{I} \partial_{\underline{2}} L_{I}-\frac{\sqrt{3}}{4} \frac{\partial_{\underline{2}} W^{2}}{W^{2}} \Sigma_{\underline{2}}^{I}\left(L_{I}-\frac{1}{12} C_{I J K} K^{J} K^{K} / H\right) .
\end{aligned}
$$

\subsection{Summary}

In this subsection we summarize for convenience the recipe to find a solution. One has to solve the system of equations given by (3.3), (3.24), (3.38) and (3.39),

$$
\begin{aligned}
\mathfrak{D}^{2} H \equiv & \partial_{\underline{1}}^{2} H+\partial_{\underline{2}}^{2}\left(W^{2} H\right)+\partial_{\underline{3}}^{2} H=0, \\
\mathfrak{D}^{2} K^{I}= & 2 \partial_{\underline{2}}\left(H W^{2} \Sigma_{\underline{2}}^{I}\right), \\
\bar{\nabla}^{2} L_{I}+ & \frac{C_{I J K}}{3 W^{2}} \partial_{\underline{2}}\left(W^{2} K^{J} \Sigma_{\underline{2}}^{K}-\frac{1}{4} H^{-1} K^{J} K^{K} \partial_{\underline{2}} W^{2}\right) \\
& +g_{I} H\left\{\frac{h_{L}}{\hat{f}}\left[\partial_{\underline{2}}\left(K^{L} / H\right)-\Sigma_{\underline{2}}^{L}\right]-\frac{4}{\sqrt{3}} \partial_{\underline{2}} \omega_{z}\right\}=0, \\
\bar{\nabla}^{2} M= & -\frac{C_{I J K}}{48 \sqrt{3} W^{2}} \partial_{\underline{2}}\left(H^{-2} K^{I} K^{J} K^{K} \partial_{\underline{2}} W^{2}\right)+\frac{C_{I J K}}{8 \sqrt{3}} H^{-1} K^{I} K^{J} \partial_{\underline{2}} \Sigma_{\underline{2}}^{K} \\
& -\frac{\sqrt{3}}{2} \Sigma_{\underline{2}}^{I} \partial_{\underline{2}} L_{I}-\frac{\sqrt{3}}{4} \frac{\partial_{\underline{2}} W^{2}}{W^{2}} \Sigma_{\underline{2}}^{I}\left(L_{I}-\frac{1}{12} C_{I J K} K^{J} K^{K} / H\right),
\end{aligned}
$$

with $\frac{h_{I}}{\hat{f}}$ and $\omega_{z}$ given by (3.31),

$$
\begin{aligned}
h_{I} / \hat{f} & \equiv L_{I}+\frac{1}{12} C_{I J K} K^{J} K^{K} / H, \\
\omega_{z} & \equiv M+\frac{\sqrt{3}}{4} L_{I} K^{I} / H+\frac{1}{24 \sqrt{3}} C_{I J K} K^{I} K^{J} K^{K} / H^{2},
\end{aligned}
$$


for the functions $H, W^{2}, K^{I}, \Sigma_{\underline{2}}^{I}, L_{I}$ and $M$ while imposing the constraints (3.20) and (3.25),

$$
\begin{aligned}
\Sigma^{I} & =4 \hat{f}^{-1} C^{I J K} h_{J} g_{K} d x^{2}, \\
2 g_{I} \Sigma_{\underline{2}}^{I} & =\hat{\nabla}^{2} \log W^{2}, \quad g_{I} K^{I}=\partial_{\underline{2}} \log W^{2} .
\end{aligned}
$$

This is still a very difficult problem, in particular because the constraint (3.45) involves the symmetric tensor $C^{I J K}$ with raised indices, which in general is not constant and cannot be written in a simple way in terms of, for instance, the functions $\frac{h_{I}}{\hat{f}}$.

To simplify the task one could assume that $C^{I J K}$ is constant, as is the case for several interesting models, in which case (3.45) and (3.44) allow to write $\Sigma_{2}^{I}$ in terms of $H, K^{I}$ and $L_{I}$. One could then proceed as follows: first choose two functions $H$ and $W^{2}$ solving equation (3.40), which amounts to choosing a base space, and subsequently solve the system of second order equations given by (3.41), (3.42) and (3.43) for $K^{I}, L_{I}$ and $M$, subject to the algebraic constraints (3.46).

Once all these functions are known, eq. (3.44) gives $\frac{h_{I}}{\hat{f}}$ and $\omega_{z}$, equations (3.2) and (3.30) can be integrated to give respectively $\chi$ and $\omega, \hat{\omega}$ is given by (3.26) and $\hat{f}$ can be obtained from the functions $\frac{h_{I}}{\hat{f}}$ using the special geometric constraint $C^{I J K} h_{I} h_{J} h_{K}=1$. At this point one has all the ingredients to write explicitly the metric (2.21), the scalar fields (2.25) and the gauge field strengths (2.24), using equations (3.17), (3.18) and (3.23).

\section{Solutions}

\subsection{Ansatz}

Assume ${ }^{14}$ for simplicity that $H$ only depends on the $\varrho$ coordinate, $H=H(\varrho)$, and that $W^{2}$ factorizes as $W^{2}=\Psi(\varrho) \Phi\left(x^{1}, x^{3}\right)$. Then from (3.40)

$$
H=\frac{a \varrho+b}{\Psi} .
$$

We will also assume $a \neq 0,{ }^{15}$ in which case one can set $a=1$ and $b=0$ by shifting and rescaling the coordinate $\varrho$, so that

$$
H=\frac{\varrho}{\Psi} .
$$

Inspired by the pure supergravity case [11] we will take $\Psi$ to be a third order polynomial in $\varrho$. In particular eq. (3.46), which implies

$$
g_{I} K^{I}=\frac{\partial_{\varrho} \Psi}{\Psi},
$$

\footnotetext{
${ }^{14}$ In what follows we will rename the coordinate $x^{2}$ to $\varrho$, both for improved readability and for the natural interpretation as "radial" coordinate.

${ }^{15}$ For pure supergravity taking $a=0$ leads to Gödel-like solutions [11]. We expect that for the $\mathrm{ST}\left[2, n_{v}+1\right]$ model we will be considering in this paper, which admits a truncation to pure supergravity, this choice would give a generalization of those solutions. We will leave the study of this possibility for future work.
} 
suggests to introduce $n_{v}+1$ polynomials

$$
\Psi^{I} \equiv \sum_{n=0}^{3} c_{n}{ }^{I} \varrho^{n}
$$

such that $\Psi=g_{I} \Psi^{I}$ and

$$
K^{I}=\frac{\partial_{\varrho} \Psi^{I}}{\Psi}
$$

Eq. (3.41) can be integrated to give

$$
\Sigma_{\underline{2}}^{I}=\frac{1}{2 \varrho}\left(-\alpha^{I}+\partial_{\varrho}^{2} \Psi^{I}\right)
$$

where $\alpha^{I}$ are integration constants, which we will take to be independent of $x^{1}$ and $x^{3}$. Eq. (3.46) implies then that $\Phi$ must be a solution of Liouville's equation

$$
\left(\partial_{\underline{1}}^{2}+\partial_{\underline{3}}^{2}\right) \log \Phi=-2 k \Phi
$$

with $k$ given by

$$
2 k=g_{I} \alpha^{I} .
$$

It is possible to choose without loss of generality $k=0, \pm 1$ and

$$
\Phi=\Phi_{(k)} \equiv \frac{4}{\left\{1+k\left[\left(x^{1}\right)^{2}+\left(x^{3}\right)^{2}\right]\right\}^{2}} .
$$

Equation (3.2) then determines $\chi$ up to a closed 1-form,

$$
d \chi=\Phi d x^{3} \wedge d x^{1} \quad \Longrightarrow \quad \chi=\chi_{(k)} \equiv \frac{2\left(x^{3} d x^{1}-x^{1} d x^{3}\right)}{1+k\left[\left(x^{1}\right)^{2}+\left(x^{3}\right)^{2}\right]}
$$

We now focus our attention on special geometric models for which the totally symmetric tensor with raised indices $C^{I J K}$ is constant. ${ }^{16}$ Comparing the expression for $\Sigma^{I}$ in (4.6) with the one in (3.45) it seems a natural choice to introduce $n_{v}+1$ first order polynomials in $\varrho, Q_{I}$, such that

$$
\frac{h_{I}}{\hat{f}}=\frac{Q_{I}}{8 \varrho}, \quad Q_{I} \equiv q_{0 I}+q_{1 I} \varrho
$$

with eq. (4.6) implying the constraints

$$
\begin{aligned}
c_{3}{ }^{I} & =\frac{1}{6} C^{I J K} g_{J} q_{1 K} \\
{c_{2}}^{I} & =\frac{1}{2}\left(\alpha^{I}+C^{I J K} g_{J} q_{0 K}\right) .
\end{aligned}
$$

One can then, after computing the functions $L_{I}$ from the definition (3.44), use equation (3.42) to obtain an expression for $\partial_{\varrho} M$. Since the expression must be the same for

\footnotetext{
${ }^{16}$ This is the case for instance when the scalar manifold is a symmetric space.
} 
each of the $n_{v}+1$ equations (one for each value of $I$ ), the following proportionality conditions must be met:

$$
\begin{aligned}
C_{I J K} c_{3}{ }^{J} c_{3}{ }^{K} & \propto g_{I} \\
4 C_{I J K} c_{3}{ }^{J}\left(\alpha^{K}-2 c_{2}{ }^{K}\right)+g_{J} c_{3}{ }^{J} q_{0 I} & \propto g_{I} \\
4 C_{I J K}\left(\alpha^{J}-2 c_{2}{ }^{J}\right) c_{1}{ }^{K}+3 g_{J} c_{1}{ }^{J} q_{0 I} & \propto g_{I} \\
2 C_{I J K} c_{1}{ }^{J} c_{1}{ }^{K}-3 g_{J} c_{0}{ }^{J} q_{0 I} & \propto g_{I} .
\end{aligned}
$$

After this, all that remains to do is to substitute $\partial_{\varrho} M$ in eq. (3.43) (we also assume for simplicity $M=M(\varrho))$ and solve the resulting algebraic equation.

\subsection{Solutions for the $\mathbf{S T}\left[2, n_{v}+1\right]$ model}

In order to find explicit solutions we will consider a specific model, namely the $\mathrm{ST}\left[2, n_{v}+1\right]$ model defined by

$$
C_{0 x y}=C^{0 x y}=\frac{\sqrt{3}}{2} \eta_{x y}
$$

where $x, y=1, \ldots, n_{v}, \eta_{x y}$ is the Minkowski $n_{v}$-dimensional metric, and the other components of $C_{I J K}$ vanish. This model reduces to pure supergravity for $n_{v}=1$ and $h^{1}=h^{0}$, and includes as a special case the STU model for $n_{v}=2$. In what follows $x$-type indices will be raised and lowered with $\eta_{x y}$ and their contraction will be denoted by a dot (e.g. $\left.g \cdot c_{1} \equiv g_{x} c_{1}^{x}\right)$. The constraints (4.12) become

$$
\begin{aligned}
c_{3}{ }^{0} & =\frac{1}{4 \sqrt{3}} g \cdot q_{1} & c_{3}{ }^{x} & =\frac{1}{4 \sqrt{3}}\left(g^{x} q_{10}+g_{0} q_{1}{ }^{x}\right) \\
c_{2}{ }^{0} & =\frac{\alpha^{0}}{2}+\frac{\sqrt{3}}{4} g \cdot q_{0} & c_{2}{ }^{x} & =\frac{\alpha^{x}}{2}+\frac{\sqrt{3}}{4}\left(g^{x} q_{00}+g_{0} q_{0}{ }^{x}\right) .
\end{aligned}
$$

The conditions (4.13) and equation (3.43) are satisfied for an arbitrary choice of gauging constants $g_{I}$ only if one of the following sets of conditions is met:

$$
\begin{aligned}
& \text { 1. } q_{00}=\frac{\sqrt{3}}{4} \frac{g_{I} c_{0} I}{\left(c_{1}{ }^{0}\right)^{2}} q_{0} \cdot q_{0}, c_{1}^{x}=\frac{\sqrt{3}}{2} \frac{g_{I} c_{0}{ }^{I}}{c_{1}{ }^{0}} q_{0}^{x}, q_{1 x}=\frac{q_{10}}{g_{0}} g_{x} \\
& \text { 2. } q_{1 x}=\frac{q_{10}}{g_{0}} g_{x}, c_{1}^{0}=g \cdot q_{0}=g \cdot c_{1}=q_{0} \cdot c_{1}=c_{1} \cdot c_{1}=g_{I} c_{0}{ }^{I}=0 \\
& \text { 3. } q_{1 x}=-\frac{q_{10}}{g_{0}} g_{x}, q_{0 I}=0 \forall I, c_{1}^{x}=0 \forall x \\
& \text { 4. } q_{1 x}=-\frac{q_{10}}{g_{0}} g_{x}, q_{0 I}=0 \forall I, c_{1}^{0}=c_{1} \cdot c_{1}=0 \\
& \text { 5. } q_{1} \cdot q_{1}=-\left(\frac{q_{10}}{g_{0}}\right)^{2} g \cdot g, g \cdot q_{1}=0, q_{0 I}=0 \forall I, c_{1}^{x}=0 \forall x \\
& \text { 6. } q_{1} \cdot q_{1}=-\left(\frac{q_{10}}{g_{0}}\right)^{2} g \cdot g, g \cdot q_{1}=0, q_{0 I}=0 \forall I, c_{1}^{0}=c_{1} \cdot c_{1}=0 \\
& \text { 7. } q_{00}=\frac{\sqrt{3}}{4} \frac{g_{I} c_{0} I}{\left(c_{1}\right)^{2}} q_{0} \cdot q_{0}, c_{1}^{x}=\frac{\sqrt{3}}{2} \frac{g_{I} c_{0} I}{c_{1}{ }^{0}} q_{0}^{x}, q_{10}=g \cdot q_{0}=g \cdot q_{1}=q_{0} \cdot q_{1}=q_{1} \cdot q_{1}=0 \\
& \text { 8. } q_{10}=c_{1}{ }^{0}=g \cdot q_{0}=g \cdot q_{1}=q_{0} \cdot q_{1}=q_{1} \cdot q_{1}=g \cdot c_{1}=q_{0} \cdot c_{1}=c_{1} \cdot c_{1}=g_{I} c_{0}{ }^{I}=0
\end{aligned}
$$

For special choices of the gauging there are some other possibilities. 
If $g \cdot g=0$ :

1. $q_{00}=\frac{\sqrt{3}}{4} \frac{g_{I} c_{0} I}{\left(c_{1}{ }^{0}\right)^{2}} q_{0} \cdot q_{0}, c_{1}^{x}=\frac{\sqrt{3}}{2} \frac{g_{I} c_{0}{ }^{I}}{c_{1}{ }^{0}} q_{0}^{x}, q_{1 x}=\beta g_{x}$

2. $q_{00}=\frac{\sqrt{3}}{4} \frac{g_{I} c_{0}^{I}}{\left(c_{1}^{0}\right)^{2}} q_{0} \cdot q_{0}, c_{1}^{x}=\frac{\sqrt{3}}{2} \frac{g_{I} c_{0}^{I}}{c_{1}{ }^{0}} q_{0}^{x}, g \cdot q_{0}=g \cdot q_{1}=q_{0} \cdot q_{1}=q_{1} \cdot q_{1}=0$

3. $c_{1}^{0}=g \cdot q_{0}=g \cdot q_{1}=q_{0} \cdot q_{1}=q_{1} \cdot q_{1}=g \cdot c_{1}=q_{0} \cdot c_{1}=c_{1} \cdot c_{1}=g_{I} c_{0}{ }^{I}=0$

If $g_{0}=0$ :

1. $q_{00}=\frac{\sqrt{3}}{4} \frac{g \cdot c_{0}}{\left(c_{1}\right)^{2}} q_{0} \cdot q_{0}, c_{1}^{x}=\frac{\sqrt{3}}{2} \frac{g \cdot c_{0}}{c_{1}{ }^{0}} q_{0}^{x}, q_{10}=0$

2. $q_{10}=c_{1}^{0}=g \cdot q_{0}=g \cdot c_{1}=q_{0} \cdot c_{1}=c_{1} \cdot c_{1}=g \cdot c_{0}=0$

If $g_{0}=g \cdot g=0$ :

1. $q_{00}=\frac{\sqrt{3}}{4} \frac{g \cdot c_{0}}{\left(c_{1}^{0}\right)^{2}} q_{0} \cdot q_{0}, c_{1}^{x}=\frac{\sqrt{3}}{2} \frac{g \cdot c_{0}}{c_{1}{ }^{0}} q_{0}^{x}$

2. $c_{1}^{0}=g \cdot q_{0}=g \cdot c_{1}=q_{0} \cdot c_{1}=c_{1} \cdot c_{1}=g \cdot c_{0}=0$

If $g_{x}=0 \forall x$ :

1. $q_{00}=\frac{\sqrt{3}}{4} \frac{g_{0} c_{0}{ }^{0}}{\left(c_{1}\right)^{2}} q_{0} \cdot q_{0}, c_{1}^{x}=\frac{\sqrt{3}}{2} \frac{g_{0} c_{0}{ }^{0}}{c_{1}{ }^{0}} q_{0}^{x}$

2. $c_{1}^{0}=q_{0} \cdot c_{1}=c_{1} \cdot c_{1}=c_{0}^{0}=0$

The function $\hat{f}$ can be computed from (4.11) using the special geometric constraint (2.1), giving

$$
\hat{f}^{-1}=\frac{\sqrt[3]{C^{I J K} Q_{I} Q_{J} Q_{K}}}{8 \varrho}=\frac{\sqrt{3}}{8 \varrho}\left[\frac{1}{2}\left(q_{00}+q_{10} \varrho\right)\left(q_{0} \cdot q_{0}+2 q_{0} \cdot q_{1} \varrho+q_{1} \cdot q_{1} \varrho^{2}\right)\right]^{1 / 3} .
$$

We are interested in particular in asymptotically anti-de Sitter solutions. Given that the line element of $\mathrm{AdS}_{5}$ (with radius $\ell$ ) can be written in standard supersymmetric form as [11]

$$
\begin{aligned}
d s^{2}= & {\left[d t+\frac{2}{\ell} \varrho\left(d z+\chi_{(k)}\right)\right]^{2}-\varrho\left(k+\frac{4}{\ell^{2}} \varrho\right)\left(d z+\chi_{(k)}\right)^{2} } \\
& -\frac{d \varrho^{2}}{\varrho\left(k+\frac{4}{\ell^{2}} \varrho\right)}-\varrho \Phi_{(k)}\left[\left(d x^{1}\right)^{2}+\left(d x^{3}\right)^{2}\right],
\end{aligned}
$$

one expects that for such solutions as $\varrho \rightarrow \infty \hat{f}$ tends to a constant and $\Psi$ diverges like $\varrho^{3}$. These conditions translate to

$$
q_{10} q_{1} \cdot q_{1} \neq 0 \quad \text { and } \quad g_{I} c_{3}{ }^{I}=\frac{1}{4 \sqrt{3}}\left(2 g_{0} g \cdot q_{1}+q_{10} g \cdot g\right) \neq 0
$$

excluding all the solutions above except the first six for arbitrary gauging. Out of these, however, only the first two are actually asymptotically AdS, at least locally, since in the other cases $\omega_{z}$ does not present the correct behavior, being proportional to $\varrho^{-1}$ (one can also check that their scalar curvature does not tend to a constant as $\varrho \rightarrow \infty)$. In the following we will analyze some properties of these two cases. 


\subsection{Case 1}

We will now analyze in detail the solutions with parameters satisfying the conditions

$$
q_{00}=\frac{\sqrt{3}}{4} \frac{g_{I} c_{0}^{I}}{\left(c_{1}\right)^{2}} q_{0} \cdot q_{0}, \quad c_{1}^{x}=\frac{\sqrt{3}}{2} \frac{g_{I} c_{0}^{I}}{c_{1}{ }^{0}} q_{0}^{x}, \quad q_{1 x}=\frac{q_{10}}{g_{0}} g_{x} .
$$

The functions $\hat{f}$ and $\Psi$ become

$$
\begin{aligned}
\hat{f}^{-1}= & \frac{\sqrt{3}}{8 \varrho}\left[\frac{1}{2}\left(\frac{\sqrt{3}}{4} \frac{g_{I} c_{0}{ }^{I}}{\left(c_{1}\right)^{2}} q_{0} \cdot q_{0}+q_{10} \varrho\right)\left(q_{0} \cdot q_{0}+2 \frac{q_{10}}{g_{0}} g \cdot q_{0} \varrho+\left(\frac{q_{10}}{g_{0}}\right)^{2} g \cdot g \varrho^{2}\right)\right]^{1 / 3}, \\
\Psi= & \frac{\sqrt{3}}{4} q_{10} g \cdot g \varrho^{3}+\left[k+\frac{\sqrt{3}}{4}\left(2 g_{0} g \cdot q_{0}+\frac{\sqrt{3}}{4} \frac{g_{I} c_{0}{ }^{I}}{\left(c_{1}\right)^{2}} q_{0} \cdot q_{0} g \cdot g\right)\right] \varrho^{2} \\
& +\left(g_{0} c_{1}{ }^{0}+\frac{\sqrt{3}}{2} \frac{g_{I} c_{0}{ }^{I}}{c_{1}{ }^{0}} g \cdot q_{0}\right) \varrho+g_{I} c_{0}{ }^{I}
\end{aligned}
$$

while $\omega_{z}$ can be obtained from eq. (3.44) after integrating $\partial_{\varrho} M$,

$$
\begin{aligned}
\omega_{z}= & \frac{3}{64}\left(q_{0} \cdot q_{0} \frac{g_{I} c_{0}{ }^{I}}{c_{1} 0} \frac{1}{\varrho^{2}}+\frac{\left(q_{10}\right)^{2}}{g_{0}} g \cdot g \varrho\right)+d \\
& +\left(q_{0} \cdot q_{0}+\frac{4}{\sqrt{3}} \frac{q_{10}}{g_{0}} c_{1}{ }^{0}\right)\left(2 g_{0}+\sqrt{3} \frac{g_{I} c_{0}{ }^{I}}{\left(c_{1}\right)^{2}} g \cdot q_{0}\right) \frac{3}{256 \varrho}
\end{aligned}
$$

where $d$ is an arbitrary constant, and $\omega$ from eq. (3.30)

$$
\omega=\left[\frac{3}{64} \frac{q_{10}}{g_{0}}\left(2 g_{0} g \cdot q_{0}+\frac{\sqrt{3}}{4} \frac{g_{I} c_{0}^{I}}{\left(c_{1}^{0}\right)^{2}} q_{0} \cdot q_{0} g \cdot g\right)-d\right] \chi_{(k)} .
$$

Since $\omega$ is of the form $\tilde{\omega} \chi$ with $\tilde{\omega}$ constant, it is always possible to reabsorb $\omega$ in $\omega_{z}$ with a shift in the $t$ coordinate, $t \rightarrow t+\tilde{\omega} z$, leading to $\omega=0$ and

$$
\begin{aligned}
\omega_{z}= & \frac{3}{64 \varrho^{2}}\left[\frac{\left(q_{10}\right)^{2}}{g_{0}} g \cdot g \varrho^{3}+\frac{q_{10}}{g_{0}}\left(2 g_{0} g \cdot q_{0}+\frac{\sqrt{3}}{4} \frac{g_{I} c_{0}{ }^{I}}{\left(c_{1}^{0}\right)^{2}} q_{0} \cdot q_{0} g \cdot g\right) \varrho^{2}\right. \\
& \left.+\left(q_{0} \cdot q_{0}+\frac{4}{\sqrt{3}} \frac{q_{10}}{g_{0}} c_{1}^{0}\right)\left(2 g_{0}+\sqrt{3} \frac{g_{I} c_{0}{ }^{I}}{\left(c_{1}^{0}\right)^{2}} g \cdot q_{0}\right) \frac{\varrho}{4}+q_{0} \cdot q_{0} \frac{g_{I} c_{0}{ }^{I}}{c_{1}{ }^{0}}\right] .
\end{aligned}
$$

The full solution is invariant under the rescaling $t \rightarrow t / \alpha, \varrho \rightarrow \alpha \varrho, q_{10} \rightarrow q_{10} / \alpha$, $c_{1}^{I} \rightarrow \alpha c_{1}^{I}, c_{0}^{I} \rightarrow \alpha^{2} c_{0}{ }^{I}$. Since we are assuming $q_{10} \neq 0$ we can use this freedom to set

$$
q_{10}=\frac{8}{\sqrt{3}} g_{0} \ell
$$

where we introduced for convenience the constant $\ell$ defined by ${ }^{17}$

$$
\ell^{3} g_{0} g \cdot g=2,
$$

so that $\hat{f} \rightarrow 1$ for $\varrho \rightarrow \infty$.

\footnotetext{
${ }^{17}$ The solutions presented here are superficially asymptotically $\mathrm{AdS}_{5}$, with $\mathrm{AdS}$ radius $|\ell|$.
} 
The line element is then

$$
\begin{aligned}
d s^{2}= & \hat{f}^{2}\left[d t+\omega_{z}\left(d z+\chi_{(k)}\right)\right]^{2} \\
& -\hat{f}^{-1}\left\{\frac{\Psi}{\varrho}\left(d z+\chi_{(k)}\right)^{2}+\frac{\varrho}{\Psi} d \varrho^{2}+\varrho \Phi_{(k)}\left[\left(d x^{1}\right)^{2}+\left(d x^{3}\right)^{2}\right]\right\},
\end{aligned}
$$

with

$$
\begin{aligned}
\hat{f}^{-3}= & \left(1+\frac{3}{32 g_{0} \ell} \frac{g_{I} c_{0}{ }^{I}}{\left(c_{1}^{0}\right)^{2}} \frac{q_{0} \cdot q_{0}}{\varrho}\right)\left(1+\frac{\sqrt{3}}{4 g \cdot g \ell} \frac{g \cdot q_{0}}{\varrho}+\frac{3}{64 g \cdot g \ell^{2}} \frac{q_{0} \cdot q_{0}}{\varrho^{2}}\right), \\
\Psi= & \frac{4}{\ell^{2}} \varrho^{3}+\left[k+\frac{\sqrt{3}}{4}\left(2 g_{0} g \cdot q_{0}+\frac{\sqrt{3}}{4} \frac{g_{I} c_{0}^{I}}{\left(c_{1}^{0}\right)^{2}} q_{0} \cdot q_{0} g \cdot g\right)\right] \varrho^{2} \\
& +\left(g_{0} c_{1}^{0}+\frac{\sqrt{3}}{2} \frac{g_{I} c_{0}^{I}}{c_{1}{ }^{0}} g \cdot q_{0}\right) \varrho+g_{I} c_{0}^{I}, \\
\omega_{z}= & \frac{2}{\ell} \varrho+\frac{3}{64 \varrho^{2}}\left[\frac{8 \ell}{\sqrt{3}}\left(2 g_{0} g \cdot q_{0}+\frac{\sqrt{3}}{4} \frac{g_{I} c_{0}^{I}}{\left(c_{1}^{0}\right)^{2}} q_{0} \cdot q_{0} g \cdot g\right) \varrho^{2}\right. \\
& \left.+\left(q_{0} \cdot q_{0}+\frac{32 \ell}{3} c_{1}^{0}\right)\left(2 g_{0}+\sqrt{3} \frac{g_{I} c_{0}{ }^{I}}{\left(c_{1}^{0}\right)^{2}} g \cdot q_{0}\right) \frac{\varrho}{4}+q_{0} \cdot q_{0} \frac{g_{I} c_{0}{ }^{I}}{c_{1}{ }^{0}}\right] .
\end{aligned}
$$

Using the parametrization (2.25) the physical scalars are given by

$$
\phi^{x}=\frac{h_{x}}{h_{0}}=\frac{h_{x} / \hat{f}}{h_{0} / \hat{f}}=\frac{8 g_{x} \ell \varrho+\sqrt{3} q_{0 x}}{8 g_{0} \ell \varrho+\frac{3}{4} \frac{g_{I} c_{0} I}{\left(c_{1}{ }^{2}\right)^{2}} q_{0} \cdot q_{0}} .
$$

The full gauge potentials are given, according to eq. (2.22), by

$$
A^{I}=-\sqrt{3} h^{I} \hat{f}\left[d t+\omega_{z}\left(d z+\chi_{(k)}\right)\right]+\hat{A}^{I},
$$

where the 4-dimensional part $\hat{A}^{I}$ can be obtained from (3.17), (3.18), (3.23),

$$
\begin{aligned}
& \hat{A}^{0}=\left(g \cdot g \ell \varrho+\frac{\sqrt{3}}{4} g \cdot q_{0}+\frac{1}{2} \frac{c_{1}^{0}}{\varrho}\right)\left(d z+\chi_{(k)}\right), \\
& \hat{A}^{x}=\left(2 g_{0} g^{x} \ell \varrho+\frac{3}{16} \frac{g_{I} c_{0}^{I}}{\left(c_{1}{ }^{0}\right)^{2}} q_{0} \cdot q_{0} g^{x}+\frac{\sqrt{3}}{4} g_{0} q_{0}{ }^{x}+\frac{\sqrt{3}}{4} \frac{g_{I} c_{0}{ }^{I}}{c_{1}{ }^{0}} \frac{q_{0}^{x}}{\varrho}\right)\left(d z+\chi_{(k)}\right),
\end{aligned}
$$

while since $h^{I}=C^{I J K} h_{J} h_{K}{ }^{18}$

$$
h^{0} \hat{f}=\frac{8 \varrho}{\sqrt{3}\left(8 g_{0} \ell \varrho+\frac{3}{4} \frac{g_{I} c_{0} I}{\left(c_{1}\right)^{2}} q_{0} \cdot q_{0}\right)}, \quad h^{x} \hat{f}=\frac{16 \varrho}{\sqrt{3}} \frac{8 g^{x} \ell \varrho+\sqrt{3} q_{0}{ }^{x}}{\left(8 g_{y} \ell \varrho+\sqrt{3} q_{0 y}\right)^{2}} .
$$

Pure supergravity is recovered by choosing $g_{x}=g_{0} \delta_{x}^{1}, q_{0 x}=q_{00} \delta_{x}^{1}$ and $q_{1 x}=q_{10} \delta_{x}^{1}$. With this choice one recovers the class of asymptotically AdS solutions of minimal gauged $\mathcal{N}=1, d=5$ supergravity found in [11].

For each value of $k$ the solutions are determined by $n_{v}+2$ parameters, $q_{0 x}, c_{1}{ }^{0}$ and $g_{I} c_{0}{ }^{I}$. The metric however only depends on the $q_{0 x}$ 's through the combinations $g \cdot q_{0}$ and $q_{0} \cdot q_{0}$, so it is always determined by four parameters, independently of the number of vector multiplets $n_{v}$.

\footnotetext{
${ }^{18}$ Note that here $h^{x} \neq \eta^{x y} h_{y}$.
} 


\subsubsection{Supersymmetric black holes}

If an event horizon exists, it must be situated in $\varrho=0$, where $\hat{f}=0$ and the supersymmetric Killing vector $\partial_{t}$ becomes null. Since $\hat{f}, H$ and $\omega_{z}$ only depend on $\varrho$, it is possible to perform a coordinate change such that

$$
\begin{aligned}
& d t=d u-H \hat{f}^{-1}\left(\hat{f}^{-1} H^{-1}-\hat{f}^{2} \omega_{z}^{2}\right)^{1 / 2} d \varrho, \\
& d z=d v-\frac{\hat{f} H \omega_{z}}{\left(\hat{f}^{-1} H^{-1}-\hat{f}^{2} \omega_{z}^{2}\right)^{1 / 2}} d \varrho,
\end{aligned}
$$

after which the metric takes the form

$$
\begin{aligned}
d s^{2}= & \hat{f}^{2} d u^{2}-\frac{2 d u d \varrho}{\left(\hat{f}^{-1} H^{-1}-\hat{f}^{2} \omega_{z}^{2}\right)^{1 / 2}}+2 \hat{f}^{2} \omega_{z} d u\left(d v+\chi_{(k)}\right) \\
& -\left(\hat{f}^{-1} H^{-1}-\hat{f}^{2} \omega_{z}^{2}\right)\left(d v+\chi_{(k)}\right)^{2}-\frac{\varrho}{\hat{f}} d \Omega_{(2, k)}^{2} .
\end{aligned}
$$

The combination $\left(\hat{f}^{-1} H^{-1}-\hat{f}^{2} \omega_{z}^{2}\right)$ tends to a constant in the limit $\varrho \rightarrow 0$, so the hypersurface $\varrho=0$ is null, and is thus a Killing horizon, if $\hat{f}^{2} \omega_{z}$ goes to zero. The only possibility to satisfy this condition without giving rise to singularities is to take the scaling limit

$$
g_{I} c_{0}^{I}=\frac{4}{\sqrt{3}} \frac{q_{00}}{q_{0} \cdot q_{0}}\left(c_{1}^{0}\right)^{2}, \quad c_{1}^{0} \rightarrow 0,
$$

in which case the functions that determine the metric become

$$
\begin{aligned}
\hat{f}^{-3} & =\left(1+\frac{\sqrt{3}}{8 g_{0} \ell} \frac{q_{00}}{\varrho}\right)\left(1+\frac{\sqrt{3}}{4 g \cdot g \ell} \frac{g \cdot q_{0}}{\varrho}+\frac{3}{64 g \cdot g \ell^{2}} \frac{q_{0} \cdot q_{0}}{\varrho^{2}}\right), \\
\Psi & =\varrho^{2}\left[\frac{4}{\ell^{2}} \varrho+k+\frac{\sqrt{3}}{4}\left(2 g_{0} g \cdot q_{0}+q_{00} g \cdot g\right)\right], \\
\omega_{z} & =\frac{2}{\ell} \varrho+\frac{3}{64 \varrho}\left[\frac{8 \ell}{\sqrt{3}}\left(2 g_{0} g \cdot q_{0}+q_{00} g \cdot g\right) \varrho+\frac{1}{2}\left(g_{0} q_{0} \cdot q_{0}+2 q_{00} g \cdot q_{0}\right)\right] .
\end{aligned}
$$

For $k=1$ these are the supersymmetric black holes of [17] with the choice (4.14), while for $k=0$ and $k=-1$ one gets a generalization of the black holes with non-compact horizon found in [11] for pure gauged supergravity.

For them to be regular, any curvature singularity should lie behind the horizon $\varrho=0$. Since the curvature scalars diverge when $\hat{f}^{-3}$ vanishes, then the zeroes of (4.40) must be negative, which translates to the conditions

$$
q_{00} g \cdot g>0, \quad q_{0} \cdot q_{0} g \cdot g>0,
$$

and either

$$
\left(g \cdot q_{0}\right)^{2}<q_{0} \cdot q_{0} g \cdot g,
$$

in which case there is only one real root, or

$$
\left(g \cdot q_{0}\right)^{2} \geq q_{0} \cdot q_{0} g \cdot g \text { and } g \cdot q_{0} g_{0}>0,
$$


in which case all roots are negative. Further constraints on the parameters come from the requirement

$$
\hat{f}^{-1} H^{-1}-\hat{f}^{2} \omega_{z}^{2}>0
$$

that also implies $H>0$.

The near horizon geometries of these black holes are themselves supersymmetric solutions and are included in the class of solutions we presented. They can be obtained from equations (4.20), (4.21) and (4.24) by taking the limit (4.39) and choosing $q_{10}=0$. They are analogous to the three near horizon geometries obtained in [20] for pure supergravity, in particular one can easily see from (4.38) that dimensional reduction along $v$ gives the geometries $\mathrm{AdS}_{2} \times S^{2}, \mathrm{AdS}_{2} \times \mathbb{H}^{2}$ or $\mathrm{AdS}_{2} \times \mathbb{E}^{2}$, and that the horizon geometry is given by a homogeneous Riemannian metric on the group manifolds $\mathrm{SU}(2)$ (in which case the metric is that of a squashed $\left.S^{3}\right), \operatorname{SL}(2, \mathbb{R})$ or $N i l$ respectively for $k=1,-1$ or 0 . The entropy density is

$$
\begin{aligned}
s_{(k)}= & \frac{\mathcal{A}_{(k)}}{3 \pi V_{(k)}}=\frac{1}{3 \pi} \frac{\sqrt{3}}{32}\left\{\sqrt{3} q_{00} q_{0} \cdot q_{0}\left[k+\frac{\sqrt{3}}{4}\left(2 g_{0} g \cdot q_{0}+q_{00} g \cdot g\right)\right]\right. \\
& \left.-\frac{3}{16}\left(g_{0} q_{0} \cdot q_{0}+2 q_{00} g \cdot q_{0}\right)^{2}\right\}^{1 / 2},
\end{aligned}
$$

where $\mathcal{A}_{(k)}$ is the area of the horizon and

$$
V_{(k)} \equiv \int \Phi_{(k)} d v \wedge d x^{1} \wedge d x^{3},
$$

so that the entropy for the $k=1$ case is $S_{(1)}=16 \pi^{2} s_{(1)}$, in agreement with the horizon area computation in [17].

\subsubsection{Conserved charges}

For $k=1$ the class of solutions we presented is asymptotically globally $\mathrm{AdS}_{5}$ according to the definition given by Ashtekar and Das in [21]. ${ }^{19}$ It is then possible to use the prescription in the same paper ${ }^{20}$ to compute the AD mass and angular momenta.

The mass is the conserved charge associated with the timelike Killing vector field

$$
V=\frac{\partial}{\partial t}+\frac{2}{\ell} \frac{\partial}{\partial z}
$$

This is the correct vector rather than the one associated with supersymmetry, since in coordinates adapted to $V$ the metric of $\mathrm{AdS}_{5}$, and in particular the metric on the conformal boundary, is written in static form. The value of the mass is

$$
\begin{aligned}
\mathcal{M}= & \frac{g_{0} \ell^{2}}{2 \sqrt{3}} g \cdot q_{0}+\frac{1}{8 g_{0} \ell} \frac{g_{I} c_{0}^{I}}{\left(c_{1}^{0}\right)^{2}} q_{0} \cdot q_{0} \\
& +\frac{3}{32 \ell}\left(q_{0} \cdot q_{0}-\frac{32 \ell}{3} c_{1}^{0}\right)\left\{2 g_{0}+\frac{g_{I} c_{0}{ }^{I}}{\left(c_{1}^{0}\right)^{2}}\left[\sqrt{3} g \cdot q_{0}+\frac{1}{\ell^{3}}\left(q_{0} \cdot q_{0}-\frac{32 \ell}{3} c_{1}^{0}\right)\right]\right\} .
\end{aligned}
$$

\footnotetext{
${ }^{19}$ See [11] for a discussion of the asymptotics of a similar class of solutions in pure gauged supergravity.

${ }^{20}$ The Ashtekar-Das paper extends to higher dimensions the original four-dimensional results obtained in an earlier paper by Ashtekar and Magnon [22]. The formalism is thus sometimes referred to as AMD (Ashtekar-Magnon-Das) formalism.
} 
Before computing the angular momenta, we perform the coordinate change

$$
z=\psi+\varphi+\frac{2}{\ell} t, \quad x^{1}=\tan \frac{\theta}{2} \cos \varphi, \quad x^{3}=\tan \frac{\theta}{2} \sin \varphi,
$$

so that

$$
d z+\chi_{(1)}=d \psi+\cos \theta d \varphi+\frac{2}{\ell} d t, \quad d \Omega_{(2,1)}^{2}=d \theta^{2}+\sin ^{2} \theta d \varphi^{2} .
$$

The angular momenta are the conserved charges associated with the Killing vectors $\partial_{\varphi}$ and $\partial_{\psi}$. They are

$$
\begin{aligned}
J_{\varphi} & =0 \\
J_{\psi} & =\frac{1}{64}\left(q_{0} \cdot q_{0}-\frac{32 \ell}{3} c_{1}^{0}\right)\left[\frac{3}{\ell^{3}} \frac{g_{I} c_{0}^{I}}{\left(c_{1}^{0}\right)^{2}}\left(q_{0} \cdot q_{0}-\frac{32 \ell}{3} c_{1}^{0}\right)+2\left(2 g_{0}+\sqrt{3} \frac{g_{I} c_{0}^{I}}{\left(c_{1}\right)^{2}} g \cdot q_{0}\right)\right] .
\end{aligned}
$$

The electric charges, defined by

$$
\mathcal{Q}_{I}=\frac{1}{8 \pi G} \int_{S_{\infty}^{3}} a_{I J} * F^{J}
$$

are

$$
\begin{aligned}
& \mathcal{Q}_{0}=\frac{1}{128}\left[g_{0}\left(q_{0} \cdot q_{0}-\frac{32 \ell}{3} c_{1}^{0}\right)\left(2 g_{0}-\sqrt{3} \frac{g_{I} c_{0}^{I}}{\left(c_{1}^{0}\right)^{2}} g \cdot q_{0}\right)-4 \frac{g_{I} c_{0}{ }^{I}}{\left(c_{1}\right)^{2}} q_{0} \cdot q_{0}\right], \\
& \mathcal{Q}_{x}=-\frac{1}{128}\left\{\left(q_{0} \cdot q_{0}-\frac{32 \ell}{3} c_{1}^{0}\right)\left[2 g_{0} g_{x}-\sqrt{3} \frac{g_{I} c_{0}{ }^{I}}{\left(c_{1}^{0}\right)^{2}}\left(g_{x} g \cdot q_{0}-g \cdot g q_{0 x}\right)\right]+\frac{16}{\sqrt{3}} q_{0 x}\right\} .
\end{aligned}
$$

It is straightforward to verify that the following BPS condition, obtained in [17] for the black hole limit of the solutions, is satisfied for all values of the parameters:

$$
\mathcal{M}-\frac{2}{\ell}|J|=4 \ell\left|\tilde{g}^{I} \mathcal{Q}_{I}\right|
$$

where we have defined

$$
\tilde{g}^{I} \equiv \lim _{\varrho \rightarrow \infty} a^{I J} g_{J} \quad \Rightarrow \quad \tilde{g}^{0}=\frac{1}{g_{0} \ell^{2}}, \quad \tilde{g}^{x}=\frac{2}{\ell^{2}} \frac{g^{x}}{g \cdot g} .
$$

\subsubsection{Static solutions}

With the choice $c_{1}{ }^{0}=\frac{3}{32 \ell} q_{0} \cdot q_{0}$ the functions $\Psi$ and $\omega_{z}$ can be expressed in a simple way in terms of $\hat{f}$,

$$
\begin{aligned}
\Psi & =\frac{4}{\ell^{2}} \varrho^{3} \hat{f}^{-3}+k \varrho^{2}, \\
\omega_{z} & =\frac{2}{\ell} \varrho \hat{f}^{-3},
\end{aligned}
$$

with $\hat{f}$ given by

$$
\hat{f}^{-3}=\frac{27}{2} \mathcal{H}_{0} \mathcal{H} \cdot \mathcal{H}
$$


where

$$
\mathcal{H}_{I} \equiv \frac{\ell}{3} g_{I}-\frac{\mathcal{Q}_{I}}{\varrho}
$$

and the $\mathcal{Q}_{I}$ 's, that for $k=1$ are the electric charges (4.56) and (4.57), are

$$
\mathcal{Q}_{0}=-\frac{32 \ell^{2}}{9} \frac{g_{I} c_{0}^{I}}{q_{0} \cdot q_{0}}, \quad \mathcal{Q}_{x}=-\frac{q_{0 x}}{8 \sqrt{3}} .
$$

The gauge potentials and scalar fields can also be written in a simple way in terms of the functions $\mathcal{H}_{I}$,

$$
A^{0}=-\frac{d t}{3 \mathcal{H}_{0}} \quad A^{x}=-\frac{2}{3} \frac{\mathcal{H}^{x}}{\mathcal{H} \cdot \mathcal{H}} d t \quad \phi^{x}=\frac{\mathcal{H}_{x}}{\mathcal{H}_{0}} .
$$

For $k= \pm 1$ it is possible to remove from the metric the cross term proportional to $d t(d z+\chi)$ by performing a simple shift in the $z$ coordinate, $z=\psi+\frac{2}{\ell k} t$, and rewrite the solutions as

$$
d s^{2}=\frac{\hat{f}^{2}}{k}\left(k+\frac{4}{l^{2}} \varrho \hat{f}^{-3}\right) d t^{2}-\frac{d \varrho^{2}}{\varrho \hat{f}\left(k+\frac{4}{l^{2}} \varrho \hat{f}^{-3}\right)}-\frac{\varrho}{\hat{f}}\left[k\left(d \psi+\chi_{(k)}\right)^{2}+d \Omega_{(2, k)}^{2}\right] .
$$

Note that these coordinates are static for $k=1$ but not for $k=-1$, since in that case the time coordinate is actually $\psi$, while $t$ is spatial. However the metric can still be rewritten in static form making first the coordinate change

$$
\psi=\tilde{\psi}-\varphi, \quad x^{1}=\tanh \frac{\theta}{2} \cos \varphi, \quad x^{3}=\tanh \frac{\theta}{2} \sin \varphi,
$$

so that

$$
d \psi+\chi_{(-1)}=d \tilde{\psi}-\cosh \theta d \varphi, \quad d \Omega_{(2,-1)}^{2}=d \theta^{2}+\sinh ^{2} \theta d \varphi^{2},
$$

followed by a second change,

$$
\tilde{\psi}=\alpha+\beta, \quad \varphi=\alpha-\beta, \quad \theta=2 \vartheta,
$$

after which it takes the form

$$
\begin{aligned}
d s^{2}= & -\hat{f}^{2}\left(-1+\frac{4}{l^{2}} \varrho \hat{f}^{-3}\right) d t^{2}-\frac{d \varrho^{2}}{\varrho \hat{f}\left(-1+\frac{4}{l^{2}} \varrho \hat{f}^{-3}\right)} \\
& -\frac{4 \varrho}{\hat{f}}\left(-\cosh ^{2} \vartheta d \beta^{2}+d \vartheta^{2}+\sinh ^{2} \vartheta d \alpha^{2}\right) .
\end{aligned}
$$

For $k=1$ one can see that substituting the chosen value of $c_{1}{ }^{0}$ in (4.54) the angular momentum vanishes as expected. In this case the three-dimensional part of the metric contained in the square brackets is just the metric of a 3-sphere, with the coordinate change

$$
\psi=\tilde{\psi}+\varphi, \quad x^{1}=\tan \frac{\theta}{2} \cos \varphi, \quad x^{3}=\tan \frac{\theta}{2} \sin \varphi,
$$

one has

$$
\left(d \psi+\chi_{(1)}\right)^{2}+d \Omega_{(2,1)}^{2}=4 d \Omega_{S^{3}}^{2}=(d \tilde{\psi}+\cos \theta d \varphi)^{2}+d \theta^{2}+\sin ^{2} \theta d \varphi^{2} .
$$


This solution was first found in [23], and can be seen as a generalization in the presence of vector multiplets of the BPS limit of the Reissner-Nördstrom-AdS 5 black hole, to which it reduces in the pure supergravity case.

For $k=0$ it is not possible to eliminate the cross term in a simple way, and the metric is

$$
d s^{2}=\hat{f}^{2} d t^{2}+\frac{4}{\ell} \frac{\varrho}{\hat{f}} d t\left(d z+\chi_{(0)}\right)-\frac{\ell^{2}}{4} \frac{\hat{f}^{2} d \varrho^{2}}{\varrho^{2}}-\frac{\varrho}{\hat{f}} d \Omega_{(2,0)}^{2} .
$$

In the pure supergravity case this reduces to a metric without free parameters and having constant curvature scalars [11]. Here this is not true in general, and only happens if

$$
\mathcal{H} \cdot \mathcal{H}=\frac{2}{\left(g_{0}\right)^{3} \ell^{3}}\left(\mathcal{H}_{0}\right)^{2},
$$

in which case the metric is the same as in the pure supergravity case, but it is still possible to have independent vector fields and non-trivial scalar fields.

\subsection{Case 2}

The solutions with

$$
q_{1 x}=\frac{q_{10}}{g_{0}} g_{x}, \quad c_{1}^{0}=g \cdot q_{0}=g \cdot c_{1}=q_{0} \cdot c_{1}=c_{1} \cdot c_{1}=g_{I} c_{0}{ }^{I}=0
$$

are almost identical to the black hole limit of the ones in subsection 4.3, given in equations $(4.40),(4.41)$ and $(4.42)$, with the additional constraint $g \cdot q_{0}=0$. However there is an additional term in the 4-dimensional gauge potentials $\hat{A}^{x}$ proportional to the constants $c_{1}{ }^{x}$, which were zero in the aforementioned limit. These constants are not completely arbitrary, being constrained by the relations $g \cdot c_{1}=q_{0} \cdot c_{1}=c_{1} \cdot c_{1}=0$.

After the rescaling (4.25) the functions determining the metric are

$$
\begin{aligned}
\hat{f}^{-3} & =\left(1+\frac{\sqrt{3}}{8 g_{0} \ell} \frac{q_{00}}{\varrho}\right)\left(1+\frac{3}{64 g \cdot g \ell^{2}} \frac{q_{0} \cdot q_{0}}{\varrho^{2}}\right), \\
\Psi & =\varrho^{2}\left(\frac{4}{\ell^{2}} \varrho+k+\frac{\sqrt{3}}{4} q_{00} g \cdot g\right), \\
\omega_{z} & =\frac{2}{\ell} \varrho+\frac{3}{64 \varrho}\left(\frac{8 \ell}{\sqrt{3}} q_{00} g \cdot g \varrho+\frac{1}{2} g_{0} q_{0} \cdot q_{0}\right),
\end{aligned}
$$

while the scalars are

$$
\phi^{x}=\frac{h_{x}}{h_{0}}=\frac{h_{x} / \hat{f}}{h_{0} / \hat{f}}=\frac{8 g_{x} \ell \varrho+\sqrt{3} q_{0 x}}{8 g_{0} \ell \varrho+\sqrt{3} q_{00}},
$$

and the gauge potentials are of the form (4.32), with

$$
\begin{aligned}
& \hat{A}^{0}=\left(g \cdot g \ell \varrho+\frac{1}{2} \frac{c_{1}^{0}}{\varrho}\right)\left(d z+\chi_{(k)}\right), \\
& \hat{A}^{x}=\left[2 g_{0} g^{x} \ell \varrho+\frac{\sqrt{3}}{4}\left(q_{00} g^{x}+g_{0} q_{0}^{x}\right)+\frac{1}{2} \frac{c_{1}^{x}}{\varrho}\right]\left(d z+\chi_{(k)}\right),
\end{aligned}
$$


and

$$
h^{0} \hat{f}=\frac{8 \varrho}{\sqrt{3}\left(8 g_{0} \ell \varrho+\sqrt{3} q_{00}\right)}, \quad h^{x} \hat{f}=\frac{16 \varrho}{\sqrt{3}} \frac{8 g^{x} \ell \varrho+\sqrt{3} q_{0}{ }^{x}}{\left(8 g_{y} \ell \varrho+\sqrt{3} q_{0 y}\right)^{2}} .
$$

For $k=1$, the mass, angular momenta and electric charges are

$$
\begin{aligned}
\mathcal{M} & =\frac{q_{00}}{2 \sqrt{3} g_{0} \ell}+\frac{3}{32 \ell} q_{0} \cdot q_{0}\left(2 g_{0}+\frac{4}{\sqrt{3} \ell^{3}} q_{00}\right), \\
J_{\varphi} & =0 \\
J_{\psi} & =\frac{q_{0} \cdot q_{0}}{16}\left(g_{0}+\frac{\sqrt{3}}{\ell^{3}} q_{00}\right), \\
\mathcal{Q}_{0} & =\frac{1}{64}\left[\left(g_{0}\right)^{2} q_{0} \cdot q_{0}-\frac{8}{\sqrt{3}} q_{00}\right], \\
\mathcal{Q}_{x} & =-\frac{1}{64}\left[g_{0} g_{x} q_{0} \cdot q_{0}+2 g \cdot g q_{00} q_{0 x}+\frac{8}{\sqrt{3}} q_{0 x}-\frac{32 \ell}{3} g \cdot g c_{1 x}\right] .
\end{aligned}
$$

Keeping into account the constraints to which the constants $q_{0 x}$ and $c_{1}{ }^{x}$ are subject, it is easy to check that the relation (4.58) is satisfied.

\section{Conclusions}

In this paper we have adapted the equations that determine the timelike supersymmetric solutions of $\mathcal{N}=1, d=5$ Abelian gauged supergravity coupled to vector multiplets to the assumption that the Kähler base space admits a holomorphic isometry. While the resulting system of equations is much more involved than in the pure supergravity case, we were able, thanks in part to the experience gained in this latter case, to obtain several supersymmetric solutions. Of these, the more interesting ones are three classes (for $k=0, \pm 1$ ) of superficially asymptotically-AdS (globally asymptotically-AdS for $k=1$ ) solutions, which are a direct generalization of the similar solutions found for pure supergravity in [11], and which include various already known solutions.

It is worth noting that the special geometric model $\mathrm{ST}\left[2, n_{v}+1\right]$ considered here admits as a special case the so-called $\mathrm{U}(1)^{3}$ model, which is just the STU model with equal gauging parameters $g_{I}$. This means that in this particular subcase our solutions can be oxidized to type-IIB supergravity as described in [24].

The solutions constructed here only have one independent angular momentum, however there are in the literature examples of supersymmetric black holes with two independent angular momenta in $\mathcal{N}=1, d=5$ Abelian gauged supergravity, both without and with vector multiplets $[25,26]$. It would be interesting to study whether less restrictive assumptions than those made in this paper could lead to solutions generalizing these black holes. ${ }^{21}$ Another possible extension of our work would be to consider more general gaugings, for instance a combination of the Abelian Fayet-Iliopoulos gauging considered here

\footnotetext{
${ }^{21}$ We have checked for instance [27] that the supersymmetric black holes of [25] can be written in the same form as the black hole solutions in the present paper or in [11], but with the function $\Phi$ not satisfying Liouville's equation and $\hat{f}$ consequently acquiring a dependence on one of the coordinates $x^{1}, x^{3}$.
} 
and non-Abelian gaugings of the scalar manifold isometries. Work along these lines is in progress [27].

\section{Acknowledgments}

The author would like to thank Tomás Ortín for his initial collaboration in this work, useful comments and discussions. This work has been supported in part by the Spanish Ministry of Science and Education grants FPA2012-35043-C02-01 and FPA2015-66793-P (MINECO/FEDER, UE) and the Centro de Excelencia Severo Ochoa Program grant SEV2012-0249.

Open Access. This article is distributed under the terms of the Creative Commons Attribution License (CC-BY 4.0), which permits any use, distribution and reproduction in any medium, provided the original author(s) and source are credited.

\section{References}

[1] R. Kallosh and T. Ortín, Killing spinor identities, hep-th/9306085 [INSPIRE].

[2] J. Kinney, J.M. Maldacena, S. Minwalla and S. Raju, An Index for 4 dimensional super conformal theories, Commun. Math. Phys. 275 (2007) 209 [hep-th/0510251] [INSPIRE].

[3] M. Berkooz, D. Reichmann and J. Simon, A Fermi Surface Model for Large Supersymmetric $A d S_{5}$ Black Holes, JHEP 01 (2007) 048 [hep-th/0604023] [INSPIRE].

[4] S.M. Hosseini, K. Hristov and A. Zaffaroni, An extremization principle for the entropy of rotating BPS black holes in $A d S_{5}$, arXiv:1705.05383 [INSPIRE].

[5] T. Ortin, Gravity and Strings, 2nd Edition, Cambridge Monographs on Mathematical Physics, Cambridge University Press, Cambridge U.K. (2015).

[6] G.W. Gibbons and S.W. Hawking, Gravitational multi-instantons, Phys. Lett. B 78 (1978) 430 [INSPIRE].

[7] G.W. Gibbons and P.J. Ruback, The Hidden Symmetries of Multicenter Metrics, Commun. Math. Phys. 115 (1988) 267 [INSPIRE].

[8] J.P. Gauntlett, J.B. Gutowski, C.M. Hull, S. Pakis and H.S. Reall, All supersymmetric solutions of minimal supergravity in five- dimensions, Class. Quant. Grav. 20 (2003) 4587 [hep-th/0209114] [INSPIRE].

[9] J.P. Gauntlett and J.B. Gutowski, General concentric black rings, Phys. Rev. D 71 (2005) 045002 [hep-th/0408122] [INSPIRE].

[10] P. Meessen, T. Ortín and P.F. Ramirez, Non-Abelian, supersymmetric black holes and strings in 5 dimensions, JHEP 03 (2016) 112 [arXiv:1512.07131] [INSPIRE].

[11] S. Chimento and T. Ortín, On timelike supersymmetric solutions of gauged minimal 5-dimensional supergravity, JHEP 04 (2017) 017 [arXiv: 1611.09383] [INSPIRE].

[12] S. Chimento and T. Ortín, On 2-dimensional Kähler metrics with one holomorphic isometry, arXiv: 1610.02078 [INSPIRE].

[13] J. Bellorín, P. Meessen and T. Ortín, All the supersymmetric solutions of $N=1, d=5$ ungauged supergravity, JHEP 01 (2007) 020 [hep-th/0610196] [INSPIRE]. 
[14] J. Bellorín and T. Ortín, Characterization of all the supersymmetric solutions of gauged $N=1, D=5$ supergravity, JHEP 08 (2007) 096 [arXiv:0705.2567] [INSPIRE].

[15] E. Bergshoeff, S. Cucu, T. de Wit, J. Gheerardyn, S. Vandoren and A. Van Proeyen, $N=2$ supergravity in five-dimensions revisited, Class. Quant. Grav. 21 (2004) 3015 [hep-th/0403045] [INSPIRE].

[16] J.P. Gauntlett and J.B. Gutowski, All supersymmetric solutions of minimal gauged supergravity in five-dimensions, Phys. Rev. D 68 (2003) 105009 [Erratum ibid. D 70 (2004) 089901] [hep-th/0304064] [INSPIRE].

[17] J.B. Gutowski and H.S. Reall, General supersymmetric AdS $S_{5}$ black holes, JHEP 04 (2004) 048 [hep-th/0401129] [INSPIRE].

[18] J.B. Gutowski and W. Sabra, General supersymmetric solutions of five-dimensional supergravity, JHEP 10 (2005) 039 [hep-th/0505185] [INSPIRE].

[19] J. Bellorín, Supersymmetric solutions of gauged five-dimensional supergravity with general matter couplings, Class. Quant. Grav. 26 (2009) 195012 [arXiv:0810.0527] [INSPIRE].

[20] J.B. Gutowski and H.S. Reall, Supersymmetric AdS $S_{5}$ black holes, JHEP 02 (2004) 006 [hep-th/0401042] [INSPIRE].

[21] A. Ashtekar and S. Das, Asymptotically Anti-de Sitter space-times: Conserved quantities, Class. Quant. Grav. 17 (2000) L17 [hep-th/9911230] [INSPIRE].

[22] A. Ashtekar and A. Magnon, Asymptotically anti-de Sitter space-times, Class. Quant. Grav. 1 (1984) L39 [INSPIRE].

[23] K. Behrndt, A.H. Chamseddine and W.A. Sabra, BPS black holes in $N=2$ five-dimensional AdS supergravity, Phys. Lett. B 442 (1998) 97 [hep-th/9807187] [INSPIRE].

[24] M. Cvetič et al., Embedding AdS black holes in ten-dimensions and eleven-dimensions, Nucl. Phys. B 558 (1999) 96 [hep-th/9903214] [INSPIRE].

[25] Z.W. Chong, M. Cvetič, H. Lü and C.N. Pope, General non-extremal rotating black holes in minimal five-dimensional gauged supergravity, Phys. Rev. Lett. 95 (2005) 161301 [hep-th/0506029] [INSPIRE].

[26] H.K. Kunduri, J. Lucietti and H.S. Reall, Supersymmetric multi-charge AdS $S_{5}$ black holes, JHEP 04 (2006) 036 [hep-th/0601156] [INSPIRE].

[27] S. Chimento, T. Ortin, P.F. Ramirez and A.V. Ruiperez, work in progress. 